Supporting Information for

\title{
Proinflammatory S10oA9 Regulate Differentiation and Aggregation of Neural Stem Cells
}

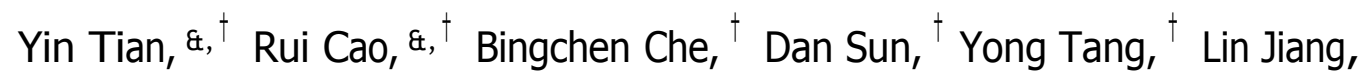

${ }^{\dagger}$ Qiao Bai, ${ }^{\dagger}$ Yonggang Liu, ${ }^{*},{ }^{\dagger}$ Ludmilla A. Morozova-Roche, ${ }^{*},{ }^{\ddagger}$ and Ce

$$
\text { Zhang } *, \dagger, \#
$$

${ }^{\dagger}$ Laboratory of Stem Cell and Tissue Engineering, Chongqing Medical University \& Institute of Photonics and Photon-Technology, Northwest University, 1 Xue Fu Avenue, Shaanxi, 710127, Xi'an, China

$\ddagger$ Department of Medical Biochemistry and Biophysics, Umeå University, 90781, Umeå, Sweden

\# Department of Pharmacy, Chongqing Three Gorges Medical College, 404120, Chongqing, China

Contains:

Supplementary Movie descriptions of Video1-3 Supplementary Figures S1-S5 


\section{Movie descriptions}

Supplementary Video 1: NSC sphere formation during suspension (left) and adherent culture (right). Notably, the PDMS surface for adherent culture is treated with laminin for merely $20 \mathrm{~min}$, which is considerably shorter than the standard protocol.

Supplementary Video 2: The NSC sphere formation process is highly dynamic, involving constant cell migrating in and out the entity.

Supplementary Video 3: NSC cells seems to group themselves based on the Dcx and Hes5 level during sphere formation and continuous culture. The cell with high Dcx level distribute only on the outer layer of the sphere, and the ones with high Hes5 stay in the core region.

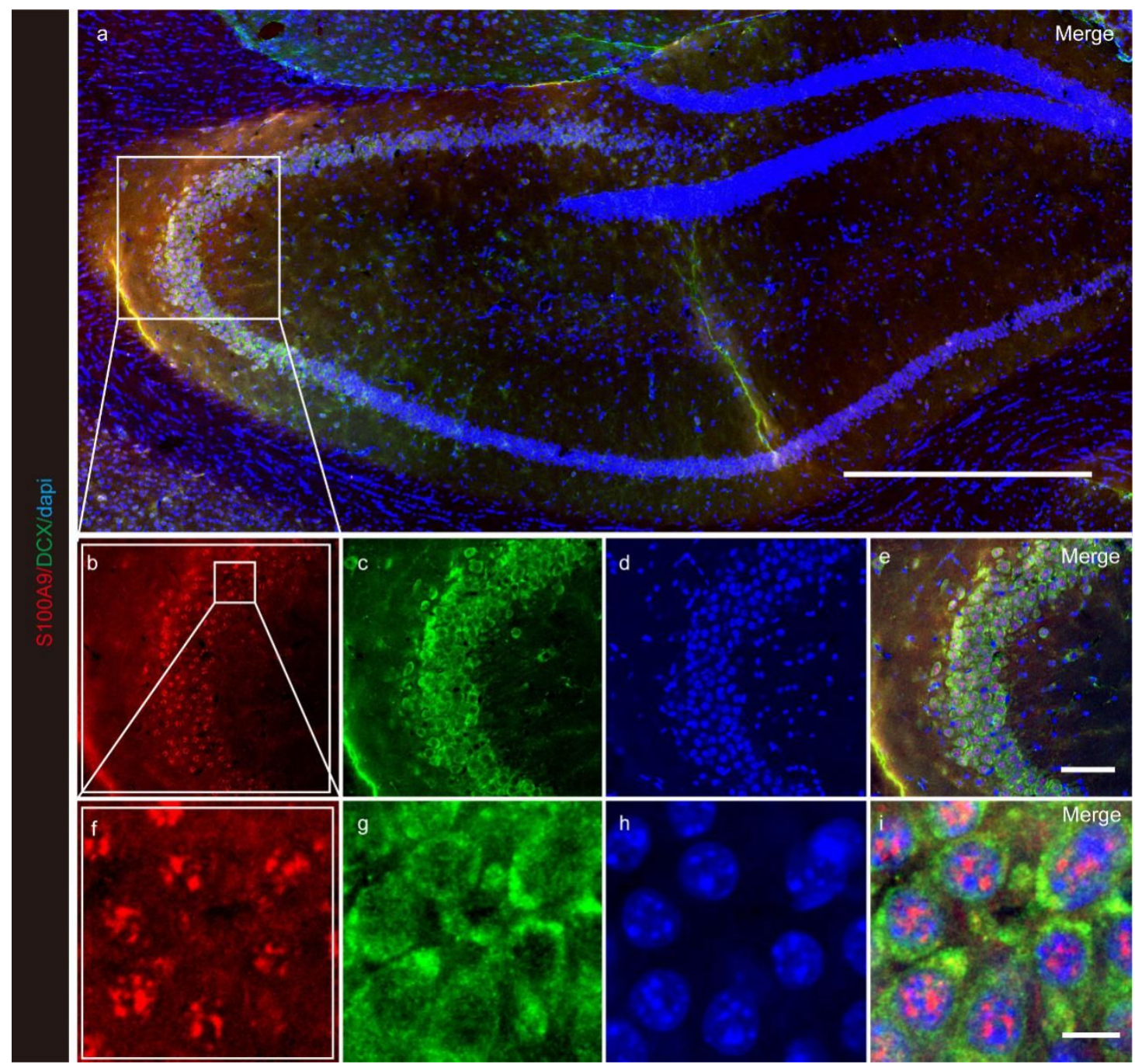

Supplementary Figure 1 S10oA9 and $\mathrm{DCX}^{+}$cells co-localization in the hippocampus. a. hippocampus large scan, Scale bars, $500 \mu \mathrm{m}$. b-e. Partial enlargement of hippocampal CA3 area, Scale bars, $65 \mu \mathrm{m}$. f-I. Show details, Scale bars, $10 \mu \mathrm{m}$. 

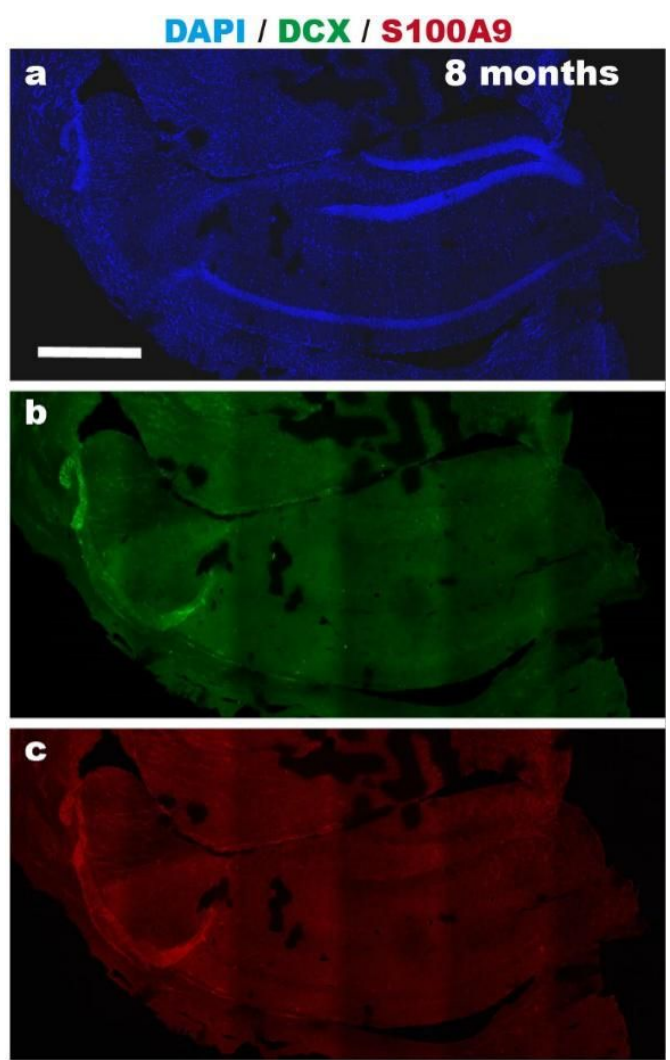

Supplementary Figure 2: Immunofluorescent staining of 8-month-old APP/PS1, mouse hippocampus with only the secondary antibodies, (a) DAPI, (b) DyLight 549 Donkey AntiRabbit IgG and (c) DyLight 488 Donkey Anti-Goat IgG. Scale bar denotes $500 \mu \mathrm{m}$.

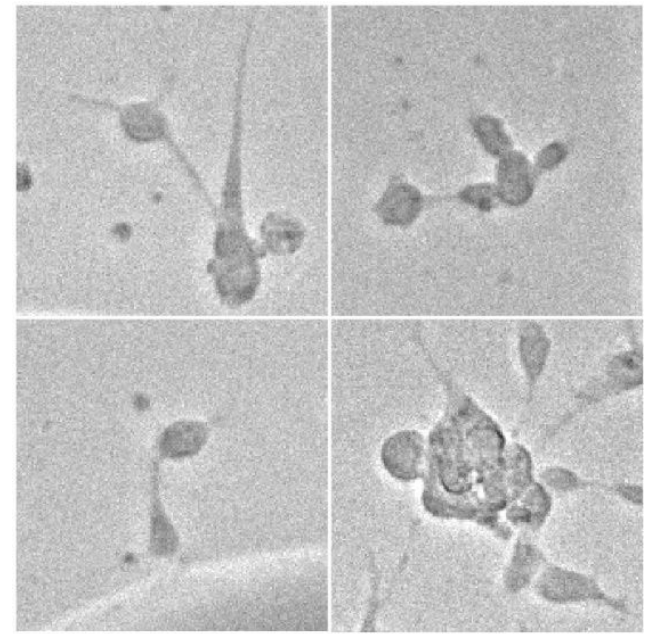

Supplementary Figure 3: Bright-field images of NSCs during sphere formation. It is demonstrated that the NSCs aggregation is directed by the axon. 


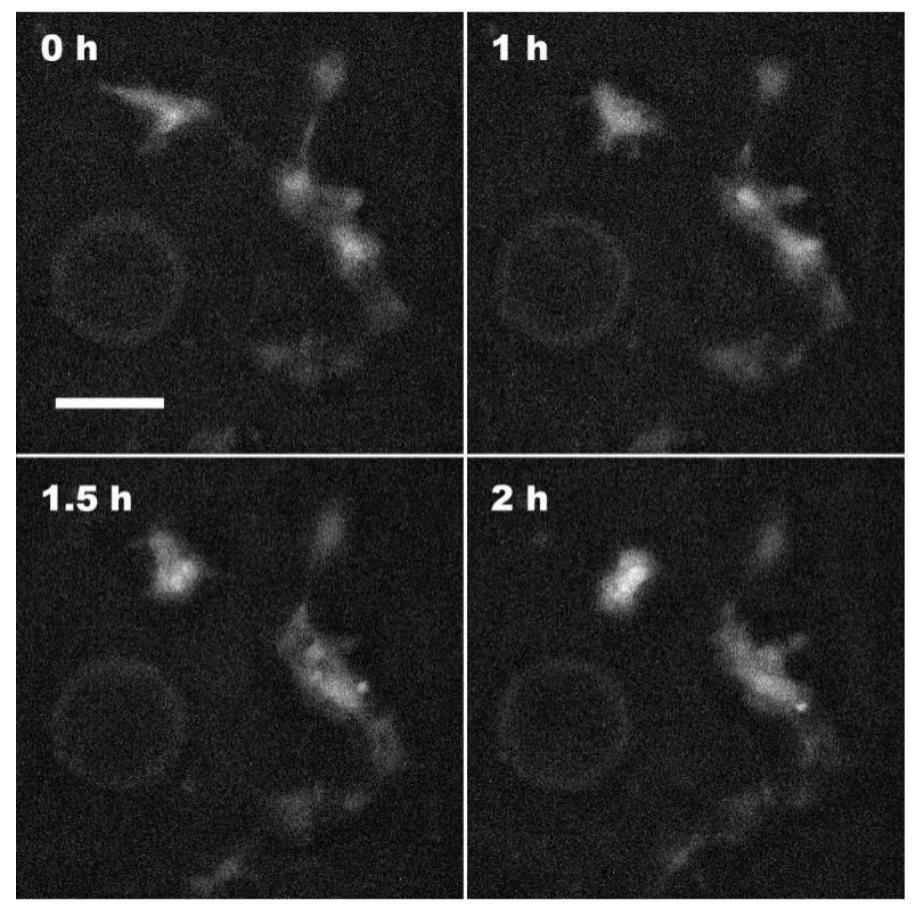

Supplementary Figure 4: Aggregation of NSCs transfected with the GFP-control vector does not bring obvious increase in the fluorescent level, excluding the effects of abnormal expression. 

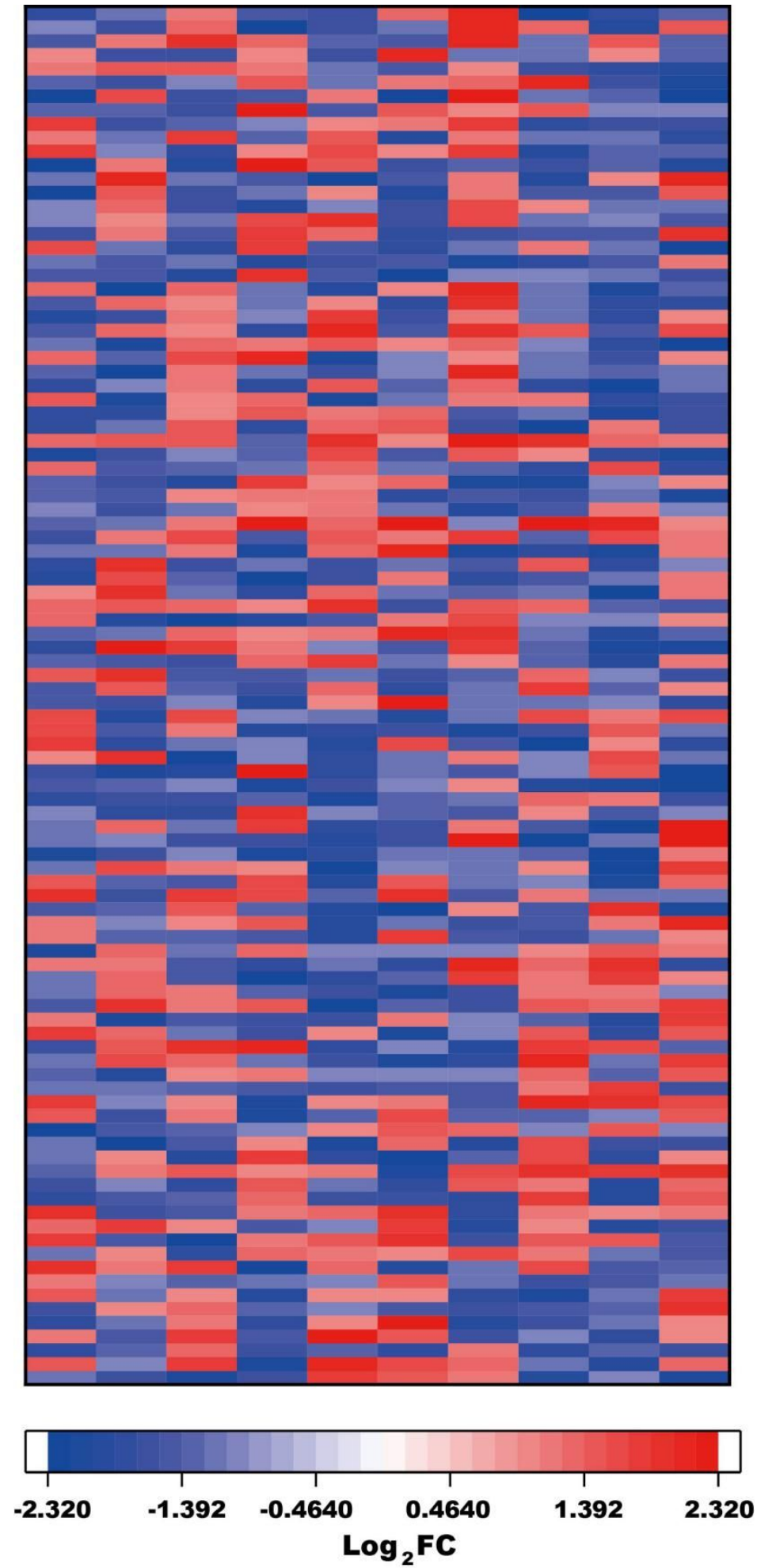

Supplementary Figure 5 Gene expression level of NSCs upon stimulation by S100A9 is normalized to the values of control samples, which are maintained in the culture medium. It is demontrated that addtion of $10 \mu \mathrm{g} / \mathrm{ml} \mathrm{S100A9}$ causes substantial amount of up- and downregulated genes. The list of gene names is shown in Supplementary Table 1. 